Supporting Information (SI)

\title{
Impacts of COVID-19 outbreak on the municipal solid waste (MSW) management: Now and beyond the pandemic
}

Poritosh Roy ${ }^{1,2}$, Amar K. Mohanty ${ }^{1,2 *}$, Alexis Wagner $^{3}$, Shayan Sharif ${ }^{4}$, Hamdy Khalil ${ }^{5}$, and Manjusri Misra ${ }^{1.2 *}$ ${ }^{1}$ School of Engineering, Thornbrough Building, University of Guelph, 50 Stone Road East, Guelph, Ontario N1G 2W1,

Canada.

${ }^{2}$ Bioproducts Discovery and Development Centre, Department of Plant Agriculture, Crop Science Building, University of Guelph, 50 Stone Road East, Guelph, Ontario N1G 2W1, Canada.

${ }^{3}$ Innovation, Science and Economic Development Canada, 235 Queen St, Ottawa, ON, K1A 0H5, Canada

${ }^{4}$ Ontario Veterinary College, University of Guelph, 50 Stone Road E., Guelph, ON, N1G 2W1 Canada,

${ }^{5}$ Woodbridge Foam Corporation, 4240 Sherwoodtowne Boulevard, Mississauga, ON, L4Z 2G6, Canada

*Corresponding author: mmisra@uoguelph.ca (M. Misra); mohanty@uoguelph.ca (A. Mohanty)

Table SI-1 Emission limits of incineration processes in different countries/regions.

\begin{tabular}{|c|c|c|c|c|c|c|c|c|}
\hline \multirow[t]{2}{*}{ Country/ region } & \multirow[t]{2}{*}{ Waste type } & \multicolumn{6}{|c|}{ Emission factors, $\mathrm{mg} / \mathrm{Nm}^{3}$} & \multirow[t]{2}{*}{ Reference } \\
\hline & & $\mathrm{CO}$ & $\mathrm{SO}_{2}$ & $\mathrm{HCl}$ & $\mathrm{NOx}$ & PM & $\mathrm{HF}$ & \\
\hline China & MSW & 80 & 80 & 50 & 250 & 20 & - & $(80,82)$ \\
\hline China & *SMW & 63 & 9.3 & 8.7 & 91 & 32 & 0.72 & $(80)$ \\
\hline China & *MSW & - & 314 & 279 & 341 & & & (81) \\
\hline Japan & MSW & 38.2 & - & 77.7 & 522.6 & 44 & - & (81) \\
\hline Japan & *MSW & - & 564 & 618 & 259 & - & - & (81) \\
\hline South Korea & MSW & 57.3 & 78.5 & 29.9 & 131.7 & 20 & - & (81) \\
\hline South Korea & *MSW & - & 65 & 263 & 604 & - & - & (81) \\
\hline US & MSW & 89 & 60 & 32.5 & 270 & 10 & - & (80) \\
\hline US & *MSW & - & 226 & - & 867 & - & - & (80) \\
\hline EU & MSW & 50 & 50 & 10 & 200 & 10 & - & (81) \\
\hline France & ${ }^{*} \mathrm{MSW}^{\wedge}$ & 43.9 & 54.0 & 23.6 & 763 & 6.24 & - & (89) \\
\hline Vietnam & *SMW & 0.065 & 0.536 & 0.0014 & - & 0.0125 & 0.0006 & (83) \\
\hline Vietnam & SMW & 250 & 250 & 50 & - & 100 & - & $(83)$ \\
\hline
\end{tabular}

PM: particulate matter; EU: European Union; US: United States; MSW: municipal solid waste; SMW: solid medical waste; HF: hydrogen fluoride; *measured data; $\wedge \mathrm{g} / \mathrm{t}$ MSW;

Table SI-2 Composition of waste streams.

\begin{tabular}{|c|c|c|c|c|c|c|c|c|c|c|c|}
\hline \multirow[t]{2}{*}{ Waste stream } & \multicolumn{10}{|c|}{ Composition, $\%$} & \multirow[t]{2}{*}{ Reference } \\
\hline & Organic & Plastic & $\begin{array}{c}\text { Paper \& } \\
\text { board }\end{array}$ & Glass & Metal & Textile & Wood & Electronic & $\begin{array}{l}\text { Building } \\
\text { material }\end{array}$ & Others & \\
\hline MSW & 64.1 & 14.1 & 7.6 & 2.8 & 1.2 & 2.2 & - & - & - & 8.0 & (90) \\
\hline MSW (LIC) & 64.0 & 8.0 & 5.0 & 3.0 & 3.0 & - & - & - & - & 17.0 & (82) \\
\hline MSW (HIC) & 28.0 & 11.0 & 31.0 & 7.0 & 6.0 & - & - & - & - & 17.0 & (82) \\
\hline MSW & 36.6 & 10.6 & 15.0 & 2.2 & 3.8 & - & 3.8 & 2.2 & 9.4 & 16.5 & (69) \\
\hline MSW & 46.5 & 20.0 & 17.0 & 4.0 & 3.0 & - & 3.0 & - & - & 6.0 & (91) \\
\hline
\end{tabular}




$\begin{array}{llllllllllll}\text { MSW } & 52.7 & 16.8 & 19.3 & 3.2 & 1.7 & - & - & - & - & 6.3 & (74) \\ \text { MSW } & 51.0 & 9.7 & 12.5 & 1.7 & 2.1 & 4.9 & 2.5 & - & - & 5.0 & (92)\end{array}$

MSW: municipal solid waste; MW: medical waste; LIC: low income countries; HIC: high income countries 